\title{
Predictors of Suicide Behavior Relapse in Pediatric Population
}

\author{
Francisco Villar ${ }^{1,2}$, Carmina Castellano-Tejedor ${ }^{2,3}$, Mireia Verge ${ }^{1}$, Bernardo Sánchez ${ }^{1}$ and \\ Tomás Blasco-Blasco² \\ ${ }^{1}$ Hospital Sant Joan de Deu (Spain). \\ 2 Universitat Autònoma de Barcelona (Spain). \\ ${ }^{3}$ Hospital Universitari Vall d'Hebron (Spain).
}

\begin{abstract}
Identifying patients at increased risk of suicide remains a challenge today. It has been reported that $10 \%$ of patients committing a suicide attempt end up dying and that both the risk and the severity of clinical symptomatology increase with the number of attempts. Within the framework of selective and indicated prevention, it is essential to identify the group of patients with an increased risk of recurrence. The objective of this study is to identify factors predicting suicide attempt relapse to improve the decision making process in the therapeutic approach to suicidal behavior. The methodology employed was a longitudinal design aimed at identifying factors, in a binary logistic regression model (stepwise), predicting the repetition of suicidal behavior among a sample of 417 participants aged between 8 and 17 years old, at the six months follow-up. A statistically significant model $\chi^{2}(3, N=417)=18.610 ; p<.001$; Nagelkerke $R^{2}=.096$ including the following factors was obtained: current diagnosis of personality disorder/maladaptive personality $O R=.806, p=.028,95 \% \mathrm{CI}[1.091,4.595]$, personal history of self-injury $\mathrm{OR}=.728, p=.043,95 \% \mathrm{CI}[1.023,4.192]$, and family history of psychopathological diagnosis $O R=.925, p=.021,95 \% \mathrm{CI}[1.151,5.530]$. Considering these results, having a diagnosis of personality disorder or maladaptive personality traits, presence or history of self-harm and family history of psychopathology draws a predictive profile of autolytic attempt recurrence during the six months after the initial intervention at the emergency room.
\end{abstract}

Received 4 January 2017; Revised 12 February 2018; Accepted 15 February 2018

Keywords: adolescence, risk factors, suicidal behavior, suicide, suicide attempt.

Suicide prevention should be a priority for the different public health systems (World Health Organization, WHO, 2014). In Spain, addressing the suicide phenomenon is a priority within the field of mental health as it is the first external cause of death in the country (Ayuso-Mateos et al., 2012). It is also the reason for the reduction of many potential years of lifespan and has significant costs for society, both economically and emotionally (Law, Yip, \& Chen, 2011). In relation to suicidal behavior, in all age groups, suicide attempts are much more prevalent than suicide, and the World Health Organization (WHO) estimates that there are 20 attempts per suicide committed (WHO, 2014). Moreover, certain groups of young people are more vulnerable than others, as it has been shown that between $20-47 \%$ of the adolescent psychiatric population attempts suicide before the age of 18 (Bursztein, \& Apter, 2009).

Suicidal behavior encompasses several behaviors. One of them is suicidal ideation, which ranges from passive ideas about dying to active thoughts about

Correspondence concerning this article should be addresssed to Francisco Villar. Hospital Sant Joan de Deu. Psychiatry and Psychology. 08950 Barcelona (Spain).

E-mail: fvillar@sjdhospitalbarcelona.org killing oneself, including planning the suicide. Another behavior is suicidal threats, understood as the verbalization of these active thoughts of killing oneself. The preparatory acts toward imminent suicidal attempt, on the other hand, would include all the preparatory acts prior to the beginning of an autolytic behavior, without actually initiating such behavior. A suicide attempt would be defined as a sequence of actions initiated by a person who, from the very moment these behaviors start, expects a lethal outcome as a consequence of the succession of such actions (The Diagnostic and Statistical Manual of Mental Disorders, DSM-5) (American Psychiatric Association, APA, 2013). Finally, a completed suicide can be considered as the deadly result of a suicide attempt.

In the context of prevention, the WHO differentiates between social or community type risk factors and individual risk factors. Among the former risks, war, natural disasters, acculturation problems (e.g., indigenous

How to cite this article:

Villar, F., Castellano-Tejedor, C., Verge, M., Sánchez, B., \& BlascoBlasco, T. (2018). Predictors of suicide behavior relapse in pediatric population. The Spanish Journal of Psychology, 21. e6. Doi:10.1017/ sjp. 2018.7 
or displaced), discrimination, loneliness, abuse, violence and conflictive relationships are highlighted. The presence of previous suicide attempts, mental disorders, harmful alcohol consumption, financial losses, chronic pain and suicide history are among the individual risk factors (WHO, 2014).

Specifically referring to the adolescent and young adult population, the most studied suicide risk factors for a first attempt include psychiatric disorders, harmful substance use, specific ethnic origins, unfavorable socioeconomic status, rural residence and being single (Pitman, Krysinska, Osborn, \& King, 2012). Another defined risk factor is family history of suicide (Brent et al., 2015). It is especially relevant to note that the main suicide risk factor described is the fact of having committed a previous attempt (Nock, Borges, Bromet, Cha et al., 2008; WHO, 2014), and that this risk increases as the number of attempts increases, with adolescents with higher recurrence rates being at a greater risk (Gould et al., 2009; Miranda et al., 2008). The percentage of attempt recurrence has been described as being around $15-16 \%$ of patients during the first year, reaching 20-25\% in the 9-year follow-up (Owens, Horrocks, \& House, 2002). Haukka and colleagues (Haukka, Suominen, Partonen, \& Lönnqvist, 2008) presented a study with 25,321 episodes of suicidal behavior that required hospitalization, generated by 18,199 people. Their results were similar to those of Owens et al., 2002, who observed a 30\% attempt recurrence in 8 years of follow-up, with the highest risk of relapse occurring during the first week after hospital discharge. The risk of mortality increased to $10 \%$ of these patients at the 8-year follow-up (Haukka et al., 2008). A similar study published a year later, with a 9-year follow-up of an adolescent population growing into adulthood, showed that $44 \%$ of the sample exhibited a suicidal behavior relapse (Groholt \& Ekeberg, 2009). Studies with adolescents with a 6-month follow-up obtained a predictive profile that included factors such as: Female gender, borderline personality disorder, previous attempts and drug use (Greenfield et al., 2008).

A more recent study by Yen et al. (2013) identified several predictors of suicidal behavior relapse in highrisk adolescents at a six-month follow-up, such as having an African American ethnicity, showing a low intensity of positive affect and aggressiveness, certain personality/temperament traits, as well as having suffered sexual abuse. These authors suggested that the cross-sectional constructs (such as affective and behavioral dysregulation) that underlie multiple psychiatric disorders may be stronger predictors of recurrent suicidal events than psychiatric diagnoses.

Thus, in order to understand the real magnitude of the problem of suicidal behavior, the correct assessment of people attending hospital emergency services after attempting suicide is essential (Kapur et al., 2008). Priority should be given to the identification of risk factors for recurrence in order to prevent suicide among a group of people who, because of their suicidal behavior, can be considered as being at high risk of suicide. Therefore, it is essential to contrast the findings in our population and enrich the research that allows us to recognize profiles of patients at a higher risk of suicide attempt relapse, both in order to prevent death, and the personal, family and social impact of these behaviors.

Taking into account these considerations, the objective of the present study is to detect predictive factors that are easily identifiable during an assessment in an emergency setting, which will allow to differentiate between the suicide attempts with good prognosis from those with bad prognosis, and to accordingly prioritize interventions for those patients at a greater risk of suicide attempt recurrence.

\section{Method}

\section{Design and setting}

This is a longitudinal study performed in the Pediatric Emergency Service of the Sant Joan de Déu Hospital (Barcelona), with an area of influence of 1,300,000 inhabitants and with 100,000 annual visits to the general emergency service.

\section{Participants}

The inclusion criteria for the sample were as follows: (1) Patients aged between 8-17 years being attended in the psychiatric emergency department of a tertiary pediatric hospital for suicide attempt and (2) patients who have exhibited a suicidal behavior different from a suicide attempt (which includes suicidal ideation, threats, or preparatory acts) with a moderate-to-high suicide risk (e.g. patients with structured suicide plans, who have carried out preparatory acts, who have verbalized their clear intention to commit a suicide attempt), according to the indications established by Nock et al. (2008). Exclusion criteria were: (1) Patients over the age of 18 years, (2) patients with cognitive or neuropsychological deficits that might hinder clinical assessment and/or the understanding of the concept of death, and (3) patients who deny any suicide intent in their behavior. That is, those that presented self-harm or parasuicidal behavior, self-inflicted harm, intoxications or other similar behaviors with anxiolytic, playful or other non-suicidal intentions.

\section{Study variables and assessment instruments}

The main variable of the study is suicide attempt relapse at the 6-month follow-up after the first discharge of 
the adolescent from the psychiatric emergency service of a pediatric third-level hospital. All patients treated at this hospital's emergency service for suicidal behavior were discharged after receiving different care procedures. One of these procedures was the creation of a security and crisis plan, in which it was specified to the parents or legal guardians that any new attempt must lead, without exception, to a new visit to the emergency services. These are the cases that were considered as relapses in the present study.

The diagnostic assessment of the patients was performed through a semi-structured clinical interview based on DSM V criteria performed by a specialist Psychiatrist or a Clinical Psychologist, who had had complementary information from previous and more complete assessments reflected in the patient's medical history, such as referral to emergency services reports, and who established the final diagnostic decision. Based on this assessment, the sociodemographic and clinical factors potentially related to the suicide attempt relapse (predictor variables) were recorded. These were:

- Gender: Male or Female.

- Age: Being under the age of 16 years vs. Being 16 years of age or over. The cutoff age of 16 years was used to establish the two levels, as it is the age at which compulsory schooling ends

- Psychopathological comorbidity in the adolescent: Yes vs. No

- Existence of a current psychopathological diagnosis of:

- Schizophrenia: Yes vs. No.

- Mood disorder: Yes vs. No.

- Anxiety disorder: Yes vs. No.

- $\quad$ Eating behavior disorder: Yes vs. No.

- Adaptive disorder: Yes vs. No.

- Personality disorder / maladaptive personality traits: Yes vs. No.

Personal history of:

- Schizophrenia: Yes vs. No.

- Mood disorder: Yes vs. No.

- Anxiety disorder: Yes vs. No.

- Eating behavior disorder: Yes vs. No.

- Adaptive disorder: Yes vs. No.

- Personality disorder / maladaptive personality traits: Yes vs. No.

- Self-harm: Yes vs. No.

- Sexual abuse: Yes vs. No.

- Background of bullying: Yes vs. No.

Family history of psychopathological diagnosis: Yes vs. No.

Personal history of suicidal behavior: Yes vs. No. Family history of suicidal behavior: Yes vs. No.

\section{Procedure}

The data collection period took place between November 2013 and December 2015, which included the six-month follow-up of all cases within the study. The collection and coding of the data based on the guidelines described above was performed on the same day of emergency care admission. All data were collected by a clinical staff member (psychologist or psychiatrist) from the Psychiatry and Psychology Department of the Sant Joan de Déu Hospital (Barcelona).

\section{Ethical aspects}

The study complies with the internal rules of the research ethics committee of the Sant Joan de Déu Hospital (Barcelona) and the Declaration of Helsinki of the World Medical Association (2013) with its successive amendments. All participants gave their informed consent freely and voluntarily for all the data collected during admission and that contained in their medical records to be used for scientific purposes, while ensuring the confidentiality, privacy and protection of their personal data.

\section{Data analyses}

In order to statistically analyze the data, the Statistical Package for the Social Sciences (SPSS), version 18 was used. To analyze the predictors of the study's main variable (patient requires new admission due to autolytic attempt during the six month follow-up), contingency tables were performed with the Chi-Square test. Subsequently, a binary logistic regression analysis (Stepwise) was performed with those variables that exhibited a statistically significant relationship with the main variable. The significance of all the tests was considered at a probability level of $5 \%$, always indicating the exact significance offered by the SPSS statistical package.

\section{Results}

\section{Descriptive Statistics}

The sample $(N=417)$ consisted of 341 women $(82.8 \%)$ and 76 men $(18.2 \%)$, aged between 8 and 17 years, $M=14.76, S D=1.56$. From this sample, 38 cases $(9.1 \%$, 35 women and 3 men) had suffered an autolytic attempt relapse at 6-month follow-up and 379 individuals $(90.9 \%)$ did not.

\section{Analysis of risk factors at the six-month follow-up (180 days)}

The factors that have shown to be significantly related to a suicide attempt relapse (379 adolescents who did not relapse vs. 38 who did) were: Comorbidity of 
psychopathological diagnosis, $\chi^{2}(1, N=417)=7.623$, $p=.010$, Eta $=.135)$; the current diagnosis of personality disorder / maladaptive personality traits, $\chi^{2}(1, N=417)=8.875, p=.007$, Eta $=.146$; the existence of a personal history of self-harm, $\chi^{2}(1, N=417)=7.889$, $p=.008$, Eta $=.138$; and the existence of a family history of psychopathological diagnosis, $\chi^{2}(1, N=417)=6.928$, $p=.010$, Eta $=.129$ (see Table 1$)$.

When the factors that showed statistically significant relationships with suicide attempt relapse at 6-month follow-up were introduced into a binary logistic regression model (stepwise), a statistically significant model, $\chi^{2}(3, N=417)=18.610 ; p<.001$; Nagelkerke $R^{2}=.096$ was obtained, which correctly classified $90.9 \%$ of the sample and included the following factors: Existence of a current diagnosis of personality disorder / maladaptive personality, $O R=.806, p=.028,95 \%$ CI [1.091, 4.595]; personal history of self-harm, $O R=.728, p=.043,95 \%$ CI [1.023, 4.192]; and a family history of psychopathological diagnosis, $O R=.925, p=.021,95 \%$ CI [1.151, 5.530]. Therefore, having a diagnosis of personality disorder or maladaptive personality traits, or having a personal history of self-harm or a family history of psychopathology were revealed as predictors of suicide attempt relapse during the first six months after the adolescent's first admission to the emergency services.

\section{Discussion}

From the results obtained in the present study, it can be observed that the majority of patients treated in the emergency services for suicidal behavior do not require a new intervention in the next six months of follow-up. However, one out of every ten patients requires emergency care again after a new suicide attempt in the referred follow-up period. Therefore, it can be concluded that within our field of intervention, the security measures, mental health follow-ups and crisis plans function correctly as protection elements in most cases. However, it is also observed that a small group of patients requires further attention as they suffer suicide attempt relapse within the first six months.

Different factors have been related to suicide attempt relapse during the mentioned period. However, the risk factors described in the literature addressing this problem are still far from conclusive and unambiguous, and many still present little predictive value. The present work aims to further deepen the study of these relationships. In order to do so, the relationship between nineteen potentially predictive variables and suicide attempt relapse during the six months after the adolescent's first admission to hospital was assessed. Both personal and socio-familial factors were considered, all of them easily identifiable during a clinical interview at an emergency service.

In the bivariate analyzes, fifteen of these variables did not show any relationship to suicide attempt relapse at the six-month follow-up. Four of these factors are especially noteworthy, as, unlike what might have been expected given that previous studies had suggested them as possible risk factors, the fact of having committed a previous attempt, the presence of suicidal behavior in relatives, depressive symptomatology and gender did not show any statistically significant relationship in the present study. Therefore, this study's results do not replicate findings such as those of Nock, Borges, Bromet, Cha et al. (2008); or the WHO (2014) that indicate as the main risk factor to have committed a previous suicide attempt. However, Yen et al. (2013), contrary to their own expectations and in line with the present study's findings, observed that having a history of previous attempts was not a predictor of a subsequent attempt during six months of follow-up. These discrepancies may be due to the disparity in the follow-up time periods used in the different studies.

Suicidal behaviors in relatives also showed no statistically significant relationship in the present study. Again, this result is similar to that reported by Yen et al. (2013), although other studies (Brent et al., 2015;

Table 1. Descriptive Statistics for the Variables Related to a Suicide Attempt Relapse.

\begin{tabular}{|c|c|c|c|c|c|c|c|}
\hline \multirow[b]{2}{*}{ Variable } & & \multicolumn{2}{|l|}{ Relapse } & \multirow[b]{2}{*}{$\chi^{2}$} & \multirow[b]{2}{*}{$d f$} & \multirow[b]{2}{*}{$p$} & \multirow[b]{2}{*}{$\eta^{2}$} \\
\hline & & Yes $n(\%)$ & No $n(\%)$ & & & & \\
\hline \multirow[t]{2}{*}{ Comorbidity } & Yes $(n=136)$ & $20(14.7 \%)$ & $116(85.3 \%)$ & 7.623 & 1 & .010 & .135 \\
\hline & No $(n=281)$ & $18(6.4 \%)$ & $263(93.6 \%)$ & & & & \\
\hline \multirow{2}{*}{$\begin{array}{l}\text { Current diagnosis of personality disorder / } \\
\text { maladaptive personality traits }\end{array}$} & Yes $(n=95)$ & $16(16.8 \%)$ & $79(83.2 \%)$ & 8.875 & 1 & .007 & .146 \\
\hline & No $(n=322)$ & $22(6.8 \%)$ & $300(93.2 \%)$ & & & & \\
\hline \multirow[t]{2}{*}{ Personal history of self-harm } & Yes $(n=154)$ & $22(14.3 \%)$ & $132(85.7 \%)$ & 7.889 & 1 & .008 & .138 \\
\hline & No $(n=263)$ & $16(6.1 \%)$ & $247(93.9 \%)$ & & & & \\
\hline \multirow{2}{*}{$\begin{array}{l}\text { Family history of psychopathological } \\
\text { diagnoses }\end{array}$} & Yes $(n=234)$ & $29(12.4 \%)$ & $205(87.6 \%)$ & 6.928 & 1 & .010 & .129 \\
\hline & No $(n=183)$ & $9(4.9 \%)$ & $174(95.1 \%)$ & & & & \\
\hline
\end{tabular}


Mittendorfer-Rutz, Rasmussen, \& Lange, 2012; Tidemalm et al., 2011) state that suicidal behavior in parents is related to an increase in suicide risk. Once more, these discrepancies could be explained through the disparity in the follow-up time periods used in the different studies, suggesting that personal history of suicide attempts and the existence of suicidal behaviors in relatives could be predictors of relapse in follow-up periods longer than 6 months after the first admission.

Another risk factor that has to date been closely related to suicidal behavior has been depression. Despite being one of Joiner, Van Orden, Witte, and Rudd (2009) "major five", depression did not show any statistically significant relationship in the present study with suicidal behavior recurrence at the six-month follow-up. The results of the present study are in line with studies indicating that severe depression or other mental illnesses do not have sufficient specificity (i.e. high rates of false positives) to guide effective preventive actions (Oquendo, Currier, \& Mann, 2006). This same result was highlighted in Greenfield et al.'s (2008) study, where authors observed that depression did not predict a future suicidal behavior relapse. Furthermore, Yen et al. (2013) did not identify depression as a predictor of attempt relapse at a six-month follow-up in a sample of adolescents, thus coinciding with the present study's results. In order to interpret these results, one must take into account Joiner (2005), who, in his interpersonal theory of suicide, provides elements that allow for a better understanding of the relationship between depression and suicidal behavior. His theoretical approach attempts to explain the process between ideation and action, between the desire to die and the attempt to commit suicide. In this line, Nock, Hwang, Sampson, and Kessler (2010) argue that depression may be a predictor of suicidal ideation, but not of planning or of attempts among those with ideation. Results of later studies follow in the same line, identifying major depressive disorder as a predictor of suicidal ideation, but no relationship is found with the transition from suicidal ideation to attempt, unlike what happens in other disorders such as Personality disorder, Behavioral disorder, Oppositional defiant disorder, Attention deficit with hyperactivity disorder and Posttraumatic stress disorder, which do predict this transition (Nock et al., 2015).

However, contrary to these studies and, therefore, also to the present study's results, other studies such as Consoli et al. (2015) and O'Connor, Smyth, and Williams (2015) determined that depression was a significant predictor of suicidal behavior relapse. Thus, further studies are needed to elucidate the relationship between depression and suicidal behavior.

Finally, although the aforementioned study by Greenfield et al. (2008) emphasized the female gender as a risk factor for relapse at six months of follow-up, in the present sample, this relationship could not be observed. Neither has any relationship between the presence of sexual abuse and risk of relapse been observed, unlike Yen et al.'s (2013) observations. Again, it is possible that these differences may be due to methodological aspects such as sample size and/or follow-up period.

Of the four variables that were related to suicide attempt relapse, diagnostic comorbidity was not part of the regression model that outlined the predictor profile. The comorbidity of psychopathological diagnoses has been mentioned as a risk factor for relapse in previous scientific literature. A large number of studies indicate that relapsing individuals present more than one psychopathological diagnosis on both the I and II axes. For example, Borges et al.'s (2010) study found that psychiatric comorbidity was a risk factor for suicidal behavior at the one-year follow-up. Possible explanations for this could be the duration of follow-up period, or the strong relationship between maladaptive personality traits and comorbidity. Therefore, adolescents who present greater comorbidity are more vulnerable due to their relationship with suicide attempt relapse. For this reason, although, according to the present study's data, it is not a predictor at the six-month follow-up, mental health services and professionals must keep this data in mind in order to guarantee greater adherence to the medium and long-term treatments.

Furthermore, the present study's results show that in our socio-cultural context and for this type of population, there is a specific profile of the adolescent who suffers suicide attempt relapse at the six-month follow-up. This profile includes the presence of a diagnosis of personality disorder or maladaptive personality traits, the existence of a family history of psychopathology and a history of self-harm. Greenfield et al.'s (2008) group found results similar to those of the present study in relation to the presence of personality disorder as a predictor of relapse. Thus, they indicated that a diagnosis of borderline personality disorder, being a woman, having performed a prior suicide attempt and drug abuse constituted a predictive profile of a new attempt at the six-month follow-up. Other similar results were found by Yen et al. (2013), where the presence of borderline personality disorder, among other factors described, was predictive of a future suicide attempt at the same follow-up period. As mentioned above, the existence of a family history of psychopathology was also part of the predictive profile found. Mittendorfer-Rutz, Rasmussem, and Wasserman (2008) reported that suicide rates and suicide attempt rates increased among adolescents with a family history of mental health and that about half of the suicide attempts were attributable to family psychopathology. 
In the same line, with a 12-month follow-up, Borges et al. (2010) identified that the presence of familial psychopathology was a predictor of relapse.

Lastly, the existence of a history of self-harm is also part of the predictive profile of relapse and, therefore, is a significant risk factor consistent with the approaches of Joiner et al.'s (2009) interpersonal theory, that explains that after a long history of selfharm, the individual increases his/her tolerance to pain and this causes him/her to progressively lose his/her fear of bodily harm, causing in turn a progressive increase of self-inflicted harm, which ultimately induces the individual to more easily perform a later suicide attempt (van Orden et al., 2010). This theory was also discussed by Nock et al. (2008). According to these authors, individuals who committed self-harm with different methods over a long period of time reported less pain during self-injury and were at an increased risk for suicide. Hence, the importance of timely detection and of not underestimating the consequences of this type of dysfunctional behavior among young people, as an early intervention can avoid future repercussions related to suicidal behavior.

The present study has a number of limitations that should be noted. The main limitation of this work is the methodology used to obtain patients' diagnosis, as it was performed through a clinical interview based on the DSM V diagnostic criteria. However, it must be considered that the intervention context was the emergency services, which hinders the administration of long assessment protocols or in-depth structured interviews. As the predictor variables considered in this study are easily assessed during a clinical interview at the emergency exam room and the recruited sample is highly representative of the population being studied, we believe that the conclusions drawn reflect the characteristics of suicidal behavior among adolescents.

Another limitation is the fact that those attempts that did not require medical attention or that were not reported by the patient, or identified by parents, teachers, or mental health professionals, are not taken into account in the present study as they are impossible to identify. Therefore, the conclusions of the study refer exclusively to those attempts that have been identified. Likewise, it would be interesting to evaluate the possible effect of the adolescent receiving some type of psychotherapeutic treatment.

In addition, another important limitation is the lack of specification of the maladaptive personality traits, as due to the methodology of the study, these characteristics could not be identified. Nevertheless, the importance of personality in the study of suicidal behavior relapse among adolescents has been observed.
Finally, while the present study, similarly to others, establishes a follow-up period of six months, considering it to be the most critical time period for a relapse episode, it would be desirable to carry out studies with a longer follow-up period that would cover at least one year, as it cannot be ruled out that this new follow-up period would yield different results. In addition, in light of the results of the present study, we believe that it would be advisable for future lines of research to further delve into the specific type of family psychopathology in cases where it is present (e.g., type of diagnosis, axis, maternal, paternal or biparental origin, etc...), as well as into the specific personality disorder of the adolescent with suicidal behavior.

The results of the present study have multiple implications and applicability, both for mental health professionals and for healthcare authorities. For the former, the identification of a predictive profile of suicide attempt relapse at a six-month follow-up helps the recommendation and guides the development of more appropriate treatment plans for these adolescents. For example, targeting these adolescents towards services of greater containment such as day hospitals, referral to programs or specific units of personality disorders, as well as establishing a greater involvement of social services and thus, a greater commitment to the family in a bid to maintain the security measures. On the other hand, knowing the predictive profile of these patients allows for a better management of the care available to the most vulnerable young people on behalf of the healthcare authorities. An example of this would be to prioritize the creation of specific programs for the early treatment of personality disorders, as placing resources in the approach of this pathology will have repercussions both on reducing the suffering of these patients and their relatives, and on the prevention of suicidal behavior among adolescents.

\section{References}

American Psychiatric Association (2013). Diagnostic and statistical manual of mental disorders $\left(5^{\text {th }}\right.$ ed.) Washington, DC: American Psychiatric Association.

Ayuso-Mateos J. L., Baca-García E., Bobes J., Giner J., Giner L., Pérez V.,... Recoms G. (2012). Recomendaciones preventivas y manejo del comportamiento suicida en España [Recommendations for the prevention and management of suicidal behavior in Spain]. Revista de Psiquiatría y Salud Mental, 5(1), 8-23.

Borges G., Nock M. K., Abad J. M. H., Hwang I., Sampson N. A., Alonso J.,... Kessler R. C. (2010). Twelve-month prevalence of and risk factors for suicide attempts in the World Health Organization World Mental Health Surveys. The Journal of Clinical Psychiatry, 71(12), 1617-1628. http:/ / doi.org/10.4088/JCP.08m04967blu

Brent D. A., Melhem N. M., Oquendo M., Burke A., Birmaher B., Stanley B.,... Mann J. J. (2015). Familial 
pathways to early-onset suicide attempt: A 5.6-year prospective study. JAMA Psychiatry, 72(2), 160-168. http://doi.org/10.1001/jamapsychiatry.2014.2141

Bursztein C., \& Apter A. (2009). Adolescent suicide: Current Opinion in Psychiatry, 22, 1-6. https://doi.org/10.1097/ YCO.0b013e3283155508

Consoli A., Cohen D., Bodeau N., Guilé J. M., Mirkovic B., Knafo A.,... Gérardin P. (2015). Risk and protective factors for suicidality at 6-month follow-up in adolescent inpatients who attempted suicide: An exploratory model. Canadian Journal of Psychiatry, 60, 27-36.

Gould M. S., Marrocco F. A., Hoagwood K., Kleinman M., Amakawa L., \& Altschuler E. (2009). Service use by at-risk youths after school-based suicide screening. Journal of the American Academy of Child \& Adolescent Psychiatry, 48(12), 1193-1201. https://doi.org/10.1097/CHI.0b013e3181bef6d5

Greenfield B., Henry M., Weiss M., Tse S. M., Guile J. M., Dougherty G.,... Harnden B.(2008). Previously suicidal adolescents: Predictors of six-month outcome. Journal of the Canadian Association of Child and Adolescent Psychiatry, 17(4), 197-201.

Groholt B., \& Ekeberg Ø. (2009). Prognosis after adolescent suicide attempt: Mental health, psychiatric treatment, and suicide attempts in a nine-year follow-up study. Suicide and Life-Threatening Behavior, 39(2), 125-136. https://doi. org/10.1521/suli.2009.39.2.125

Haukka J., Suominen K., Partonen T., \& Lönnqvist J. (2008). Determinants and outcomes of serious attempted suicide: A nationwide study in Finland, 1996-2003. American Journal of Epidemiology, 167(10), 1155-1163. https://doi.org/10.1093/aje/kwn017

Joiner Jr., T. E., Van Orden K. A., Witte T. K., \& Rudd M. D. (2009). The Interpersonal Theory of Suicide: Guidance for working with suicidal clients. Washington, DC: American Psychological Association.

Joiner T. E. (2005). Why people die by suicide. Cambridge, MA: Harvard University Press.

Kapur N., Murphy E., Cooper J., Bergen H., Hawton K., Simkin S.,... Owens D. (2008). Psychosocial assessment following self-harm: Results from the multi-centre monitoring of self-harm project. Journal of Affective Disorders, 106(3), 285-293. https://doi.org/10.1016/ j.jad.2007.07.010

Law C. K., Yip P. S. F., \& Chen Y. Y. (2011). The economic and potential years of life lost from suicide in Taiwan, 1997-2007. Crisis, 32(3), 152-159. https:/ / doi.org/ 10.1027/0227-5910/a000070

Miranda R., Scott M., Hicks R., Wilcox H. C., Munfakh J. L. H., \& Shaffer D. (2008). Suicide attempt characteristics, diagnoses, and future attempts: Comparing multiple attempters to single attempters and ideators. Journal of the American Academy of Child and Adolescent Psychiatry, 47(1), 32-40. https:/ / doi.org/10.1097/chi.0b013e31815a56cb

Mittendorfer-Rutz E., Rasmussen F., \& Lange T. (2012). A life-course study on effects of parental markers of morbidity and mortality on offspring's suicide attempt. PLoS ONE, 7(12), e51585. https://doi.org/10.1371/journal. pone. 0051585
Mittendorfer-Rutz E., Rasmussen F., \& Wasserman D. (2008). Familial clustering of suicidal behavior and psychopathology in young suicide attempters. Social Psychiatry and Psychiatric Epidemiology, 43(1), 28-36. https://doi.org/10.1007/s00127-007-0266-0

Nock M. K., Borges G., Bromet E. J., Cha C. B., Kessler R. C., \& Lee S. (2008). Suicide and suicidal behavior. Epidemiologic Reviews, 30(1), 133-154. https:/ / doi.org/10.1093/epirev/mxn002

Nock M. K., Hwang I., Sampson N. A., \& Kessler R. C. (2010). Mental disorders, comorbidity and suicidal behavior: Results from the National Comorbidity Survey Replication. Molecular Psychiatry, 15(8), 868-876. https:/ / doi.org/10.1038/mp.2009.29

Nock M. K., Ursano R. J., Heeringa S. G., Stein M. B., Jain S., Raman R., ... Kessler R. C. (2015). Mental disorders, comorbidity and pre-enlistment suicidal behavior among new soldiers in the U.S. Army: Results from the Army Study to Assess Risk and Resilience in Service members (Army STARRS). Suicide E Life-Threatening Behavior, 45, 588-599. https://doi.org/10.1111/sltb.12153

O'Connor R. C., Smyth R., \& Williams J. M. G. (2015). Intrapersonal positive future thinking predicts repeat suicide attempts in hospital-treated suicide attempters. Journal of Consulting and Clinical Psychology, 83(1), 169-176. https://doi.org/10.1037/a0037846

Oquendo M. A., Currier D., \& Mann J. J. (2006). Prospective studies of suicidal behavior in major depressive and bipolar disorders: What is the evidence for predictive risk factors? Acta Psychiatrica Scandinavica, 114(3), 151-158. https://doi.org/10.1111/j.1600-0447.2006.00829.x

Owens D., Horrocks J., \& House A. (2002). Fatal and non-fatal repetition of self-harm. The British Journal of Psychiatry, 181(3), 193-199. https://doi.org/10.1192/ bjp.181.3.193

Pitman A., Krysinska K., Osborn D., \& King M. (2012). Suicide in young men. The Lancet, 379, 2383-2392. https: / / doi.org/10.1016/S0140-6736(12)60731-4

Tidemalm D., Runeson B., Waern M., Frisell T., Carlström E., Lichtenstein P., \& Långström N. (2011). Familial clustering of suicide risk: A total population study of 11.4 million individuals. Psychological Medicine, 41(12), 2527-2534. https: / / doi.org/10.1017/ S0033291711000833

van Orden K. A., Witte T. K., Cukrowicz K. C., Braithwaite S. R., Selby E. A., \& Joiner Jr., T. E. (2010). The interpersonal theory of suicide. Psychological Review, 117(2), 575-600. https:/ / doi.org/10.1037/ a0018697

World Health Organization (WHO). (2014). Preventing suicide: A global imperative. Retrieved from $\mathrm{WHO}$ website http:/ /apps.who.int/iris/bitstream/10665/131056/ 1/9789241564779_eng.pdf?ua=1\&ua=1

Yen S., Weinstock L. M., Andover M. S., Sheets E. S., Selby E. A., \& Spirito A. (2013). Prospective predictors of adolescent suicidality: 6-month post-hospitalization follow-up. Psychological Medicine, 43(5), 983-993. https:/ / doi.org/10.1017/S0033291712001912 